\title{
Análisis del sistema de producción y caracterización microestructural para evaluar el comportamiento mecánico de chasis de vehículo fabricado por extrusión.

\begin{abstract}
Analysis of the production system and microstructural characterization to evaluate the mechanical behavior of vehicle chassis manufactured by extrusion.
\end{abstract}

Luis Antonio Chica Castro. ${ }^{1}$, Juan Carlos Rocha Hoyos. ${ }^{2}$, Javier Martínez Gomez. ${ }^{3}$ \& Patricio Cabascango Camuendo. ${ }^{4}$

\begin{abstract}
.
DOI: https://doi.org/10.33262/cienciadigital.v3i1.349

The vehicles have been a fundamental part in the movement of people and objects to different places saving time. Aluminum has played an important role in the development of new technologies for the construction of vehicles and this is where auto parts play an important role. The production of these has grown from basic processes to automated processes, which increases the economy and development of the country. That is why this work aims to analyze the production of aluminum by extrusion and characterize microstructurally alloys 6061 and 6063 used in the manufacture of auto parts. The methodology used was to analyze the systems and production lines of auto parts, perform tensile tests, compression and hardness of the alloys 6061 and 6063 respectively and finally characterize microstructurally the alloys that make up this study in order to obtain which is the most recommended to carry out the process of extruding auto parts. The conclusion is that the automated production line can reduce production times and in turn strengthens the productivity also that 6063 alloy is the material used for the manufacturing process of auto parts since they apply less compression efforts, preventing lose the resistance to corrosion. Además, se produce mayor resistencia después del proceso de conformado debido
\end{abstract}

\footnotetext{
${ }^{1}$ Universidad Internacional SEK Quito, Ecuador, 1chica.mdm@uisek.edu.ec

${ }^{2}$ Universidad Internacional SEK Quito, Ecuador, carlos.rocha@uisek.edu.ec

${ }^{3}$ Universidad Internacional SEK Quito, Ecuador, javier.martinez@uisek.edu.ec

${ }^{4}$ Universidad Internacional SEK Quito, Ecuador, ccabascango.mdm@uisek.edu.ec
} 
a las formas del $\mathrm{Fe}_{3} \mathrm{SiAl}_{12}$ después de la deformación incrementando así la vida útil de la autoparte.

Keywords: Auto parts, extrusion, aluminum, alloys, intermetallic compound

\section{Resumen.}

Los vehículos han sido parte fundamental en el desplazamiento de personas y objetos a diferentes lugares ahorrando tiempo. El aluminio ha tenido un papel importante en el desarrollo de nuevas tecnologías para la construcción de los vehículos y es allí donde las autopartes juegan un papel importante. La producción de las mismas ha crecido pasando de procesos básicos a procesos automatizados con lo cual aumenta la economía y desarrollo del país. Es por ello que este trabajo tiene como objetivo analizar la producción de aluminio por extrusión y caracterizar microestructuralmente las aleaciones 6061 y 6063 utilizadas en la fabricación de autopartes. La metodología utilizada fue analizar los sistemas y líneas de producción de autopartes, realizar ensayos de tracción, compresión y dureza de las aleaciones 6061 y 6063 respectivamente y por último caracterizar microestructuralmente las aleaciones que conforman este estudio con el fin de obtener cual es la más recomendada para realizar el proceso de extrusión de autopartes. Como conclusión se tiene que la línea de producción automatizada puede disminuir los tiempos de producción y a su vez fortalece la productividad también que la aleación 6063 sea el material utilizado para el proceso de fabricación de autopartes ya que se aplican menores esfuerzos de compresión, evitando que se pierda la resistencia a la corrosión. Además, se produce mayor resistencia después del proceso de conformado debido a las formas del $\mathrm{Fe}_{3} \mathrm{SiAl}_{12}$ después de la deformación incrementando así la vida útil de la autoparte.

Palabras claves: Autopartes, extrusión, aluminio, aleaciones, compuesto intermetálico

\section{Introducción.}

La industria automotriz es parte de la base fundamental en el desarrollo mundial. $\mathrm{Su}$ crecimiento está vinculado a actividades productivas y también al traslado de personas y de bienes. La fabricación de autopartes es muy importante para el desarrollo del país debido a que promueve el PIB, genera empleo, resalta entre los demás sectores de la economía y fortalece la competitividad del comercio exterior.

Es por ello que la industria de autopartes no escapa de una constante evolución. Este desarrollo evolutivo es consecuencia de evaluaciones de los procesos de producción, derivado de los estudios y análisis de mercado, y de los procesos de conformado utilizando los mejores materiales, para cada autoparte, que puedan soportar las cargas o esfuerzos que garanticen un mejor rendimiento. 
Para el desarrollo de la producción de autopartes para vehículos es necesario comprender los sistemas de producción. El sistema de producción por transformación se desarrolla cuando todas las operaciones se organizan para lograr una situación ideal, cuando la economía de fabricación favorece a la producción, es decir cuando la demanda de un producto determinado es elevada y cuando el producto se realice a gran escala. Este tipo de sistema ofrece obreros especializados y semiespecializados y los costos de producción son relativamente bajos (Iplacex, 2018).

En Ecuador la producción de autopartes es muy limitada ya que la industria está conformada por pequeñas y medianas empresas que tienen bajo nivel de tecnología, lo que constituye para este eslabón de la cadena, un grave obstáculo para conseguir un mayor grado de productividad y de competitividad. Por consiguiente, sería preciso armonizar los esfuerzos hacia la producción especializada de partes a utilizarse en el armado de unos pocos modelos, especialmente en los segmentos de automóviles compactos de bajo precio que son los de mayor demanda local, sin tener que importar un volumen cada vez mayor de partes y piezas como tradicionalmente se ha venido haciendo, buscando además que se modifiquen las políticas de precios del sector empresarial, para que se beneficie también el consumidor nacional con vehículos de calidad y garantía a menor precio que los importados (Bardellini, 2005).

Los materiales a utilizar son muy importantes porque garantizaran un mejor trabajo en el conformado de piezas. La selección de los mismos debe destacar sus propiedades mecánicas, eléctricas y térmicas que ayuden a mejorar el proceso de deformación, pero también garanticen una mayor vida útil cuando estén sometidos los esfuerzos de ingeniería sobre los mismos. Es por ello el uso del aluminio por sus bajos costos, gran maquinabilidad a la hora de trabajarlos y sobre todo las piezas fabricadas tienen menor peso y poseen alta resistencia a la corrosión en ambientes drásticos.

Así mismo, la producción de autopartes de aluminio ayudara aún más al proceso de conformado ya que las fuerzas que se requieren son menores y se pueden obtener piezas de diferente geometría debido a la característica planteada anteriormente. Por ello, es necesario aplicar un análisis de la microestructura de dichas piezas para poder determinar cuáles son los parámetros de conformado a utilizar y poder así obtener piezas de alta calidad. Se analizaron aleaciones de aluminio 6061 y 6063 debido a que pueden ser deformadas muy fácilmente, a través de la extrusión, y que pueden soportar la corrosión generada, como también son materiales de gran accesibilidad ya que se producen en el país.

El proceso de extrusión consiste en generar una pieza conformada por fuerzas que se ejecutan a un tocho por medio de un punzón y que serán deformadas a través de un dado o matriz. La velocidad de este proceso está directamente relacionada con las presiones, características dimensionales del tocho y, sobre todo, por la composición química de ese material. Existen procesos de extrusión directa, indirecta y por impacto. Dependiendo del diseño de la pieza 
final se recurre a un proceso de extrusión. Para el caso de la fabricación de autopartes se recurre a la extrusión directa si las piezas las conforman las ballestas o estructura inferior del chasis y si es por extrusión por impacto las estructuras que conforman las partes laterales del chasis.

Por tal motivo, este trabajo tiene como objetivo analizar la producción y caracterización del aluminio por extrusión para uso en autopartes. Se evaluaron los diferentes procesos y líneas de producción por extrusión, se determinó el proceso de producción y tipo de pieza a fabricar por extrusión, se caracterizó la microestructura de las aleaciones de aluminio y se determinó el comportamiento de esta autoparte de aluminio mediante el análisis de la microestructura con el fin de obtener los parámetros de conformado más acorde en el proceso de extrusión.

\section{Metodología.}

Esta investigación requirió de una investigación exploratoria, pasando por una investigación experimental y concluyendo con una investigación explicativa.

Para el desarrollo se utilizaron 24 probetas que correspondieron a 12 probetas para cada tipo de aleación de aluminio, incluyendo 1 muestra patrón para cada tipo. (ver Tabla 1)

Tabla 1. Probetas para cada tipo de ensayo.

\begin{tabular}{ccccc}
\hline $\begin{array}{c}\text { Tipo de } \\
\text { Aleación }\end{array}$ & Muestra Patrón & $\begin{array}{c}\text { Ensayo de } \\
\text { Tracción }\end{array}$ & $\begin{array}{c}\text { Ensayo de } \\
\text { Dureza }\end{array}$ & $\begin{array}{c}\text { Ensayo de } \\
\text { Compresión }\end{array}$ \\
\hline Aleación 1 & 1 & 4 & 3 & 4 \\
\hline Aleación 2 & 1 & 4 & 3 & 4 \\
\hline Total & 2 & 8 & 6 & 8 \\
\hline
\end{tabular}

Elaborado por: Grupo de Investigación

Se detalla la metodología experimental que sirvió para llegar a la consecución de los objetivos del proyecto:

\section{Análisis de los distintos sistemas y líneas de producción para la fabricación de autopartes.}

Se analizaron los sistemas de producción para la fabricación de productos de aluminio por extrusión con la finalidad de obtener los parámetros de producción para realizar la fabricación de autopartes. Un sistema de producción es cualquier actividad que produzca algo. Sin embargo, se definió de manera más formal como aquello que toma un insumo y lo transforma en una salida o producto con valor inherente (Sipper \& Bulfin, 1998).

También se hizo un análisis del factor de productividad que no es más que la relación o cociente entre bienes o servicios producidos $\mathrm{u}$ ofrecidos entre los medios utilizados 
(Cuatrecasas, 2011). Para los procesos de extrusión la productividad es una función de indicador de gestión que mejorará la producción de autopartes tomando en cuenta cual es la línea de producción que más se adecuada a estas variables y a su vez establecer recomendaciones de mejoras con el fin de obtener productos con los estándares de los sistemas integrados de gestión, basados en las normas ISO9000, ISO14000 e ISO18000, y así obtener un desarrollo sostenible y sustentable de la nación.

\section{Determinación de las diferentes aleaciones de aluminio.}

Se tomaron los diferentes materiales provenientes de reconocidas empresas del país dedicas a la producción de aluminio estructural con el fin de obtener las probetas para los ensayos destructivos y para la caracterización del material.

La utilización de aluminio se debe a que se pueden fabricar campanas, baúles, acabado brillante, entradas de aire y parachoques (Metal handbook Volumen 2, 1989). En el área de extrusión la mayor parte el mercado es tomado por la serie 6000 AlMgSi (TALAT 1302, 1994). Los materiales se tomaron gracias a un estudio detallado sobre los materiales más usados en el proceso de extrusión de autopartes como también por ser de mayor accesibilidad en el mercado ecuatoriano con el fin de encontrar los parámetros del proceso y así obtener los resultados más acordes y afines a la realidad.

\section{Ensayo de tracción.}

El ensayo de tracción se realizó bajo las recomendaciones de la norma ASTM E8M-16a ("Standard Test Methods for Tension Testing of Metallic Materials"). Se ensayaron 8 probetas que corresponden a 4 para cada tipo de aleación. Las dimensiones de cada probeta fueron tomadas según la norma establecida para este ensayo, como se muestra en la Figura 1.

Figura 1. Dimensiones de las probetas siguiendo la norma establecida para el ensayo de tracción. (A) Diseño según norma y (B) Probeta fabricada de aluminio.

(A)

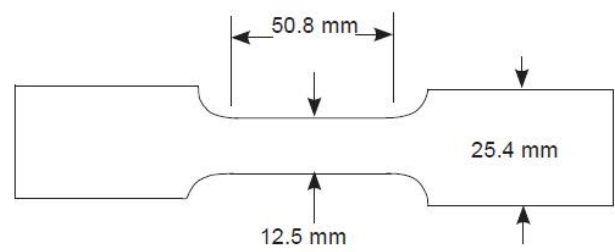

(B)

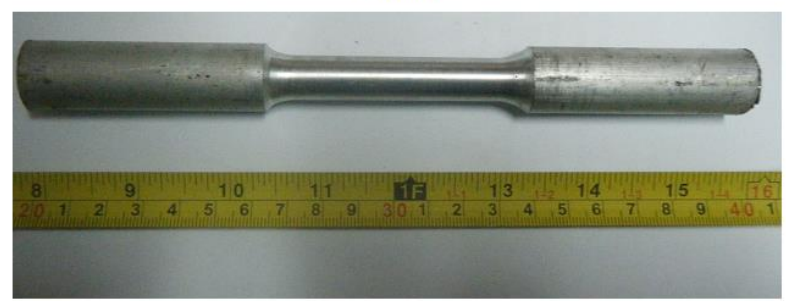


En este ensayo el material se deforma hasta la rotura mediante la aplicación de una fuera uniaxial a lo largo del eje principal (Guerrero, Davila y Pontón, 2011).

\section{Ensayos de dureza.}

Este ensayo se realizó con el apoyo de un durómetro Rockwell basándose en la norma ASTM E18-03 ("Norma para la realización del ensayo de dureza rockwell"), tomando mediciones de dureza en diferentes zonas para así tener un valor más exacto de las aleaciones de aluminio. El ensayo se realizó a un total de 6 muestras, 3 muestras para cada tipo de aleación de aluminio.

La dureza es una medida de resistencia del material a la deformación plástica localizada. La dureza rockwell puede utilizar diferentes escalas provenientes de las combinaciones entre penetradores y cargas, lo cual permite ensayar cualquier metal o aleación desde el más duro al más blando (Callister, 2014). Esto se puede observar en la Figura 2.

Figura 2. Técnicas de ensayo de dureza rockwell.
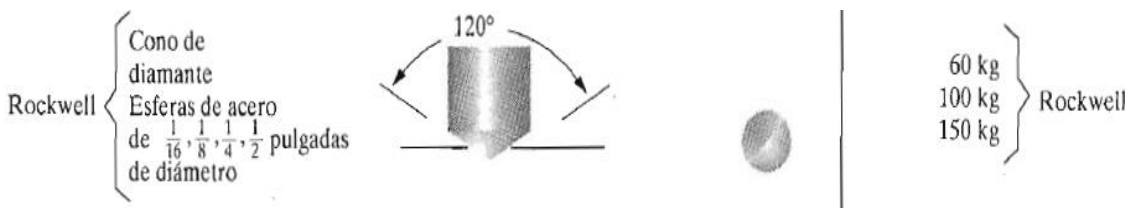

Para esta investigación se utilizó el ensayo de dureza rockwell B con una carga de $100 \mathrm{Kg}$ y un penetrador de bola $1 / 16$ pulg durante $15 \mathrm{seg}$.

\section{Ensayo de compresión.}

Se realizó este ensayo a 8 probetas, 4 para cada tipo de aleación. Se basó en la norma ASTM E-9 ("Standard Test Methods for Compression Testing of Metallic Materials"). Las dimensiones de las probetas están mostradas en la Figura 3 son tomadas de la norma establecida.

Figura 3. Dimensiones de las probetas siguiendo la norma establecida para el ensayo de compresión. (A) Diseño según norma y (B) Probeta fabricada de aluminio.

(A)

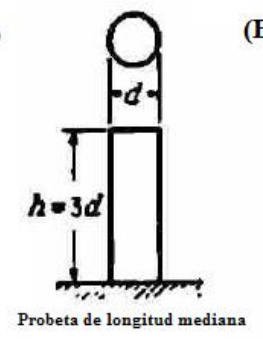

(B)

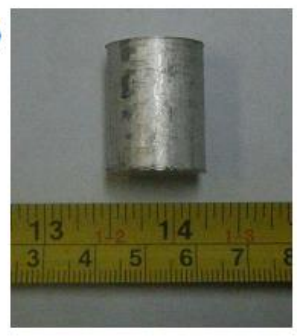


La finalidad de este ensayo es poder determinar el comportamiento mecánico de las distintas aleaciones, con respecto a fuerzas de compresión, y correlacionarlas con las propiedades mecánicas del ensayo de tracción. Estos resultados ayudaran al comportamiento de las aleaciones cuando están sometidas a las cargas de la extrusora.

El esfuerzo y deformación ingenieriles en un ensayo de compresión se definen con respecto a la longitud y área originales del espécimen (Nañez, 1998).

\section{Caracterización microestructural.}

Para la preparación metalográfica de las probetas patrón y deformadas por extrusión se realizó el siguiente procedimiento basado en la norma ASTM E-3 ("Standard guide for preparation of metallographic specimens"). La observación de la microestructura se hizo mediante el microscopio óptico (MO) para identificar: fases, morfología, tamaño, proporción y microdefectos provenientes de la deformación.

Los resultados de este ensayo determinaran cuál de las aleaciones es la más acorde a los planteamientos y exigencias en el proceso de extrusión para así obtener piezas de autopartes con alta garantía de calidad, respetando el medio ambiente y valorando la seguridad y protección laboral.

\section{Resultados Y Discusión}

\section{Análisis de los sistemas y líneas de producción para la fabricación de autopartes.}

Es importante resaltar el proceso de fabricación de autopartes de aluminio por extrusión como se ilustra en la Figura 4.

La producción de autopartes por medio del proceso de extrusión es muy parecida al proceso de extrusión general. Las empresas dependen de contratistas para garantizar material y piezas para el desarrollo de la producción lo cual la hacen ser sistemas de transformación. Este proceso de conformado es realizado a través de técnicas de extrusión directa o por impacto y se diferencias dependiendo del diseño de la autoparte. Cuando el producto final son ballestas, que conforman el sistema de suspensión, el proceso se realiza a través de la extrusión directa, pero si el producto son componentes del chasis el proceso es a través de la extrusión por impacto.

La contratista provee el aluminio para ser fundidos en los hornos, luego se realiza la colada continua para pasar al primer proceso de conformado como es la laminación en caliente. Aquí los esfuerzos son menores y se obtienen mayores deformaciones. Posteriormente se realiza la laminación en frio donde al final la lámina es bobinada para después pasar por el proceso de corte en la troqueladora la cual otorga la primera forma de la autoparte. De aquí los productos semi-terminados pasan al horno de recocido debido a la laminación en frio ya que 
esto produce un endurecimiento por deformación. El objetivo del recocido es otorgar ductilidad al semi-producto para que estos sean deformados en la extrusora sin ningún problema. La extrusión por impacto hace que la pieza adquiera la forma final de la autoparte.

Figura 4. Proceso de producción de autopartes a través del proceso de extrusión de pletinas de aluminio.

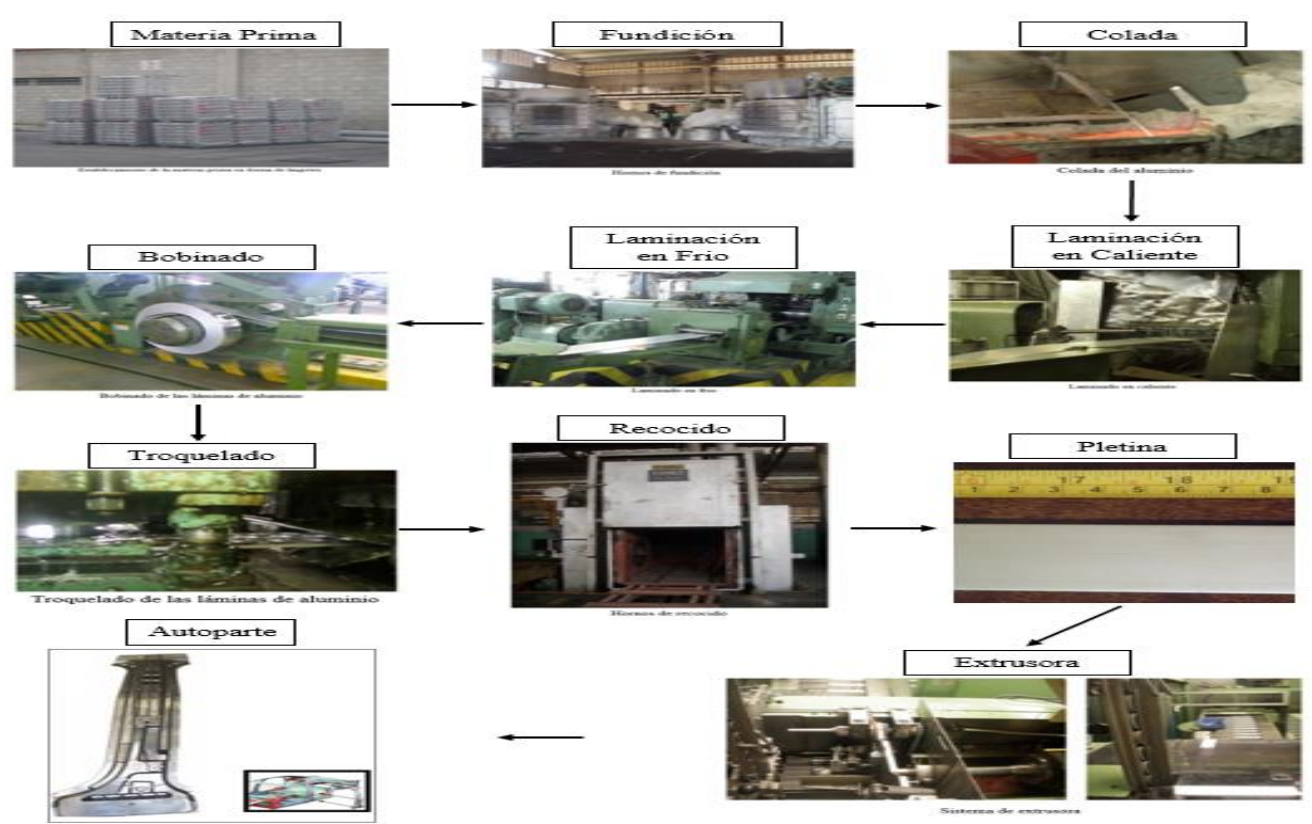

Los parámetros operacionales del proceso de extrusión de autopartes tienen un significado importante ya que va a depender de la composición química de la aleación de aluminio como se detalla en la Tabla 2. Se destaca la influencia de la composición de la materia prima en razón de la velocidad del proceso. Según (Navas y col, 2017) las aleaciones de la serie 6000 no deben de exceder de un contenido de 0,70 de porcentaje en peso de $\mathrm{Mg}$, porque reduce la velocidad de extrusión y perjudica la productividad. Esto se refiere a una relación estequiométrica entre el magnesio y el silicio la cual arroja un valor de 1,73. Si esta relación es menor a 1,73 la velocidad es mayor y si la relación es mayor a 1,73 entonces la velocidad es menor.

Es muy importante determinar la velocidad porque en ella depende la cantidad de producción. La presión va a depender de la dimensión del tocho y del diámetro del dado o matriz por lo tanto es una propiedad física de diseño.

Tabla 2. Parámetros del proceso de extrusión para piezas de aluminio. 


\begin{tabular}{|c|c|c|c|c|c|c|c|c|}
\hline \multicolumn{9}{|c|}{ PARAMETROS DEL PROCESO DE EXTRUSIÓN } \\
\hline \multirow{3}{*}{ Aleación } & \multirow{3}{*}{$\begin{array}{l}\text { Comp. } \\
\text { Química }\end{array}$} & \multirow{3}{*}{$\begin{array}{c}\text { Relación } \\
\frac{\% M g}{\% S i}\end{array}$} & \multirow{2}{*}{\multicolumn{2}{|c|}{ VELOCIDAD }} & \multicolumn{4}{|c|}{ PRESION } \\
\hline & & & & & \multicolumn{2}{|c|}{ LONGITUD } & \multicolumn{2}{|c|}{ DIAMETRO DEL DADO } \\
\hline & & & $\mathrm{R}<1,73$ & $\mathrm{R}>1,73$ & MENOR & MAYOR & MENOR & MAYOR \\
\hline ----- & - -.--- & -.--- & MAYOR & MENOR & MENOR & MAYOR & MAYOR & MENOR \\
\hline
\end{tabular}

Una forma de incrementar la productividad del proceso de extrusión de autopartes es solventando el tiempo de traslado de los productos semiterminados entre cada estación de trabajo. Para ello se plantea una línea de producción automatizada como se muestra en la Figura 5. Se muestra un sistema automatizado para aumentar la producción a través de una línea de producción automatizada.

Esta línea estará colocada de forma continua y unida a través de cintas transportadoras las cuales deben soportar pesos y temperaturas variables. Estas cintas transportadoras también contarán con un variador de frecuencia para manejar distintas velocidades según sea la estación de trabajo.

En el área de recocido la velocidad debe ser lenta para que del tiempo suficiente para poder llegar a la temperatura de homogenización antes de salir del horno. Al igual ocurre en la zona de ventilación donde se debe controlar la velocidad de enfriamiento con el fin de cambiar las propiedades del material. Cuando la pieza sale de la zona de extrusión, se propone el uso de brazos robótico para la selección y empaquetado de las autopartes en una paletizadora. Por supuesto esto genera grandes inversiones de capital, pero el tiempo de recuperación del mismo será corto ya que se aumentará la productividad de la organización.

Figura 5. Línea de producción automatizada para la fabricación de autopartes de aluminio por extrusión.

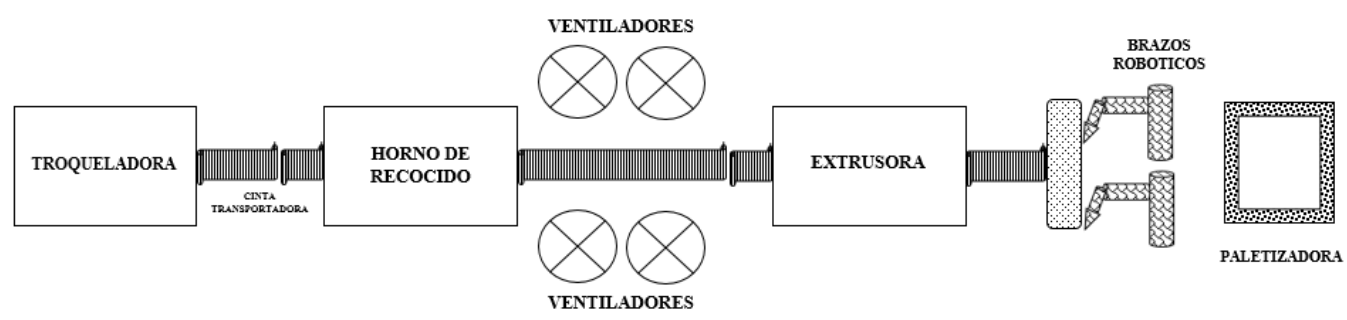

\section{Determinación de las diferentes aleaciones de aluminio.}

Para la fabricación de autopartes es necesario analizar los materiales más adecuados para el mejor funcionamiento del vehículo. Se toma en consideración las aleaciones de aluminio por su bajo peso y resistencia a la corrosión y al desgaste. Las aleaciones de la serie 6000 son las mejores para el proceso de conformado por extrusión por lo cual se toman las aleaciones 
6061 y 6063, debido a su gran uso en la industria y por su fácil accesibilidad en el mercado, con el fin de evaluar y obtener la más adecuada para estos procesos de manufactura.

Para conocer los componentes químicos de las aleaciones a estudiar fue necesario realizar un análisis por medio del espectrómetro de masa con el fin de estipular el porcentaje en que se encuentran presentes los elementos aleantes, los resultados se muestran en la Tabla 3.

Tabla 3. Composición química de las aleaciones 6061 y 6063.

\begin{tabular}{|c|c|c|c|c|c|c|c|c|c|c|c|}
\hline Aleacion & $\% \mathrm{Al}$ & $\% \mathrm{Mg}$ & $\% \mathrm{Si}$ & $\% \mathrm{Fe}$ & $\% \mathrm{Cr}$ & $\% \mathrm{Ti}$ & $\% \mathrm{Zn}$ & $\% \mathrm{Mn}$ & $\% \mathrm{Cu}$ & $\% \mathrm{Ga}$ & $\% \mathrm{~V}$ \\
\hline 6061 & 98,96 & 0,4522 & 0,385 & 0,166 & 0,017 & 0,010 & 0,0013 & 0,0013 & 0,001 & 0,008 & 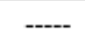 \\
\hline 6063 & 97,81 & 0,879 & 0,582 & 0,295 & 0,167 & 0,034 & 0,0045 & 0,0247 & 0,167 & ב-. & 0,0068 \\
\hline
\end{tabular}

La influencia de los elementos aleantes es muy importante porque radica en que pueden determinar el comportamiento de la aleación a fuerzas externas o mejor a los procesos de conformado. Esto se puede observar en la relación $\frac{\% M g}{\% S i}$ ya que puede afectar el comportamiento de la productividad en función de la velocidad del proceso de extrusión. Para ir más allá de este análisis es necesario comprender el funcionamiento de cada elemento dentro de la aleación y para ello se presenta la Tabla 4 donde se detalla los elementos químicos principales en función de las propiedades del material.

Se observa como la influencia del $\mathrm{Si}, \mathrm{Fe}, \mathrm{Mn}$ y $\mathrm{Cu}$ aumenta la resistencia mecánica. El Mg otorga mayor ductilidad. La presencia de $\mathrm{Si}$, Fe, Ti y $\mathrm{Zn}$ aumenta la al agrietamiento en caliente. Los elementos como $\mathrm{Mn}$ y $\mathrm{Cu}$ aumentan la dureza de la aleación. Estas propiedades mecánicas son importantes de resaltar porque estas aleaciones pasarán por los ensayos de tracción y compresión, donde estarán involucradas esfuerzos de trabajo, pero sobre todo pasaran por procesos de conformado por extrusión las cuales se deben garantizar que no produzcan concentradores de tensiones y así aumentar la vida útil de las autopartes.

Tabla 4. Influencia de la composición química en las aleaciones de la serie 6000 en función de las propiedades del material. 


\begin{tabular}{|c|c|c|c|c|c|c|c|}
\hline Propiedades $\quad \begin{array}{c}\text { Elementos } \\
\text { Químicos }\end{array}$ & $\% \mathrm{Mg}$ & $\% \mathrm{Si}$ & $\% \mathrm{Fe}$ & $\% \mathrm{Ti}$ & $\% \mathbf{Z n}$ & $\% \mathrm{Mn}$ & $\% \mathrm{Cu}$ \\
\hline Resistencia Mecánica & ----- & Mayor & Mayor & ----- & ----- & Mayor & Mayor \\
\hline Dureza & ---- & 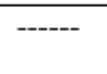 & ---- & ----- & 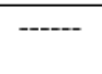 & Mayor & Mayor \\
\hline Ductilidad & Mayor & ----- & Menor & ----- & ----- & Notable & ----- \\
\hline Resistencia en Caliente & ----- & Mayor & Mayor & Mayor & Mayor & - & Menor \\
\hline Resistividad Térmica & Menor & Mayor & ----- & ------ & ----- & $-\cdots$ & ----- \\
\hline Resistencia a la Corrosión & Mayor & ----- & ----- & ----- & ---- & Igual & Menor \\
\hline
\end{tabular}

Comparando estos resultados de la Tabla 4 con los resultados de composición química de la Tabla 3 se puede observar claramente que la aleación 6063 posee mayor cantidad de elementos químicos que resaltan la resistencia a la deformación por lo cual puede indicarse que afectaran al comportamiento mecánico a esfuerzos de tracción y comprensión, comparados con el comportamiento de la aleación 6061.

\section{Ensayo de tracción.}

En la Tabla 5 se puede observar los valores de esfuerzo de fluencia y máximo de las probetas sometidas a tracción de la aleación 6061. Se tomaron todos los valores obtenidos y se realizó un promedio para compararlo con las probetas de la aleación 6063 ensayadas bajo cargas de tracción.

Tabla 5. Esfuerzos de fluencia y máximo de las probetas de aleación 6061 con las respectivas deformaciones provenientes del ensayo de tracción.

\begin{tabular}{|c|c|c|c|c|}
\hline \multicolumn{5}{|c|}{ Aleación 6061} \\
\hline & \multicolumn{2}{|c|}{ Esfuerzos ( $\sigma)$ en MPas } & \multicolumn{2}{|c|}{ Deformación ( $\varepsilon$ ) en mm } \\
\hline Probetas & $\sigma_{\text {Fluencia }}$ & $\sigma_{\text {Máximo }}$ & $\varepsilon_{\text {ofluencia }}$ & $\varepsilon_{\text {бmáximo }}$ \\
\hline $\mathbf{P}_{\mathrm{ATl}}$ & 54,22 & 264,14 & 0,041 & 0,171 \\
\hline $\mathbf{P}_{\mathrm{AT} 2}$ & 47,30 & 235,00 & 0,034 & 0,183 \\
\hline $\mathbf{P}_{\text {AT3 }}$ & 66,04 & 255,90 & 0,065 & 0,140 \\
\hline $\mathbf{P}_{\mathrm{AT} 4}$ & 62,75 & 250,99 & 0,073 & 0,144 \\
\hline Promedio & 57,58 & 251,51 & 0,053 & 0,160 \\
\hline
\end{tabular}

También se realizaron ensayos de tracción para las probetas de aleación 6063 las cuales obtuvieron esfuerzos de fluencia y máximo con las respectivas deformaciones como se muestra en la Tabla 6. Se tomaron todos los valores obtenidos y se realizó un promedio para compararlo con las probetas de la aleación 6061 ensayadas bajo cargas de tracción. En la Figura 6 se puede observar la gráfica esfuerzo-deformación promedio de los ensayos de tracción realizados a las probetas de aleación 6061 y 6063 respectivamente. 
Tabla 6. Esfuerzos de fluencia y máximo de las probetas de aleación 6063 con las respectivas deformaciones provenientes del ensayo de tracción.

\begin{tabular}{|c|c|c|c|c|}
\hline \multicolumn{5}{|c|}{ Aleación 6063} \\
\hline & \multicolumn{2}{|c|}{ Esfuerzos ( $\sigma)$ en MPas } & \multicolumn{2}{|c|}{ Deformación ( $(\varepsilon)$ en mm } \\
\hline Probetas & $\sigma_{\text {Fluencia }}$ & $\sigma_{\text {Máximo }}$ & $\varepsilon_{\text {ofluencia }}$ & $\varepsilon_{\text {бmáximo }}$ \\
\hline $\mathbf{P}_{\mathrm{BTl}}$ & 191,82 & 262,52 & 0,00725 & 0,08000 \\
\hline $\mathrm{P}_{\mathrm{BT} 2}$ & 191,51 & 271,29 & 0,00751 & 0,06395 \\
\hline $\mathbf{P}_{\mathrm{BT} 3}$ & 199,16 & 284,52 & 0,00655 & 0,06910 \\
\hline $\mathbf{P}_{\mathrm{BT} 4}$ & 192,29 & 255,36 & 0,00564 & 0,04678 \\
\hline Promedio & 193,70 & 268,42 & 0,00674 & 0,06496 \\
\hline
\end{tabular}

Figura 6. Gráfica esfuerzo-deformación promedio del ensayo de tracción de las probetas de aleación 6061 y 6063 respectivamente.

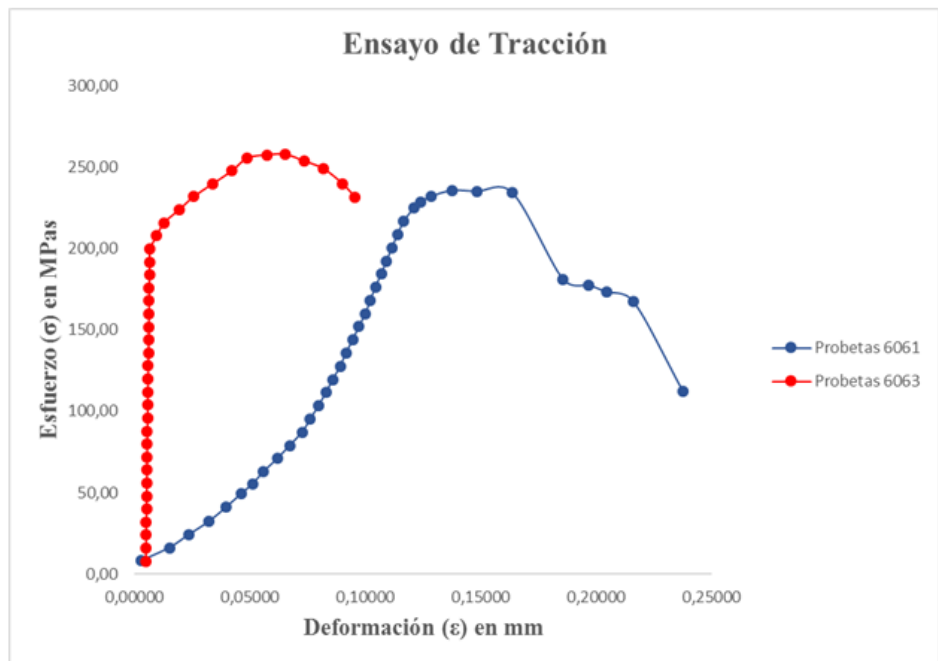

Se observan las dos curvas de esfuerzo-deformación de las aleaciones 6061 y 6063 respectivamente. La curva de la aleación 6061 posee mayor deformación plástica la cual hace que la fractura se prolongue en el tiempo. La curva de la aleación 6063 posee menor deformación haciéndola más resistente a los esfuerzos.

Esto podría tener una explicación y es debido a que las dislocaciones presentes dentro de las fases no tienen tanto espacio para movilizarse, es decir que los tamaños de grano son pequeños, o también que puedan existir compuestos intermetálicos que se formaron gracias a la unión de los elementos metálicos que conforman las aleaciones. Más factible es la presencia de estos compuestos intermetálicos que generan cierta resistencia a los esfuerzos y por ende no se produzca la deformación plástica.

\section{Ensayo de dureza.}


En la Tabla 7 se puede observar los valores de dureza rockwell B promedio de las probetas de aleación 6061 y 6063 respectivamente. Todos los valores de medición de dureza se encuentran en el ANEXO II.

Tabla 7. Dureza rockwell B promedio de las probetas de leación 6061 y 6063 respectivamente.

\begin{tabular}{rccc} 
& \multicolumn{3}{c}{ DUREZA ROCKWELL B } \\
\cline { 2 - 4 } Aleación & Probeta 1 & Probeta 2 & Probeta 3 \\
\hline $\mathbf{6 0 6 1}$ & 50 & 48 & 49 \\
\hline $\mathbf{6 0 6 3}$ & 63 & 63 & 64 \\
\hline
\end{tabular}

Las aleaciones 6063 poseen mayor dureza, comparada con la aleación 6061, por lo cual hace que sea más resistente a esfuerzos mecánicos y esto se comprueba con el resultado de la gráfica esfuerzo-deformación de la Figura 6. Esta resistencia se debe al endurecimiento por solución sólida de los elementos aleantes dentro de la aleación ya que hay mayor cantidad de solutos dentro del solvente de la aleación 6063 haciendo más endurecible. Este endurecimiento por solución sólida va de la mano con la formación de compuestos intermetálicos entre los elementos químicos.

\section{Ensayo de compresión.}

En la Tabla 8 se puede observar los valores de esfuerzo de fluencia y máximo de las probetas sometidas a compresión de la aleación 6061. Se tomaron todos los valores obtenidos y se realizó un promedio para compararlo con las probetas de la aleación 6063 ensayadas bajo cargas de compresión.

Tabla 8. Esfuerzos de fluencia y máximo de las probetas de aleación 6061 con las respectivas deformaciones provenientes del ensayo de compresión

\begin{tabular}{|c|c|c|c|c|}
\hline \multicolumn{5}{|c|}{ Aleación 6061} \\
\hline \multirow[b]{2}{*}{ Probetas } & \multicolumn{2}{|c|}{ Esfuerzos ( $\sigma$ ) en MPas } & \multicolumn{2}{|c|}{ Deformación $(\varepsilon)$ en mm } \\
\hline & $\sigma_{\text {Fluencia }}$ & $\sigma_{\text {Máximo }}$ & $\varepsilon_{\text {ofluencia }}$ & $\varepsilon_{\text {omáximo }}$ \\
\hline $\mathbf{P}_{\mathrm{ACl}}$ & 83,25 & 989,52 & 0,00951 & 0,69276 \\
\hline $\mathbf{P}_{\mathrm{AC2} 2}$ & 258,64 & 851,36 & 0,10545 & 0,69276 \\
\hline $\mathbf{P}_{\mathrm{AC} 3}$ & 269,84 & 945,24 & 0,12687 & 0,84256 \\
\hline $\mathbf{P}_{\mathrm{AC} 4}$ & 253,97 & 911,25 & 0,11146 & 0,79369 \\
\hline Promedio & 216,43 & 924,34 & 0,08832 & 0.75544 \\
\hline
\end{tabular}

También se realizaron ensayos de compresión para las probetas de aleación 6063 las cuales obtuvieron esfuerzos de fluencia y máximo con las respectivas deformaciones como se muestra en la Tabla 9. Se tomaron todos los valores obtenidos y se realizó un 
promedio para compararlo con las probetas de la aleación 6061 ensayadas bajo cargas de compresión.

Tabla 9. Esfuerzos de fluencia y máximo de las probetas de aleación 6063 con las respectivas deformaciones provenientes del ensayo de compresión

\begin{tabular}{|c|c|c|c|c|}
\hline \multicolumn{5}{|c|}{ Aleación 6063} \\
\hline & \multicolumn{2}{|c|}{ Esfuerzos ( $\sigma)$ en MPas } & \multicolumn{2}{|c|}{ Deformación ( $(\varepsilon)$ en mm } \\
\hline Probetas & $\sigma_{\text {Fluencia }}$ & $\sigma_{\text {Máximo }}$ & $\varepsilon_{\text {ofluencia }}$ & $\varepsilon_{\text {бmáximo }}$ \\
\hline $\mathbf{P}_{\mathrm{BCl}}$ & 115,13 & 435,26 & 0,00250 & 0,36000 \\
\hline $\mathrm{P}_{\mathrm{BC} 2}$ & 158,47 & 406,49 & 0,02130 & 0,29500 \\
\hline $\mathbf{P}_{\mathrm{BC} 3}$ & 152,32 & 398,27 & 0,02498 & 0,31049 \\
\hline $\mathbf{P}_{\mathrm{BC} 4}$ & 177,70 & 382,25 & 0,01080 & 0,18354 \\
\hline Promedio & 150,91 & 405,57 & 0,01486 & 0.28726 \\
\hline
\end{tabular}

En la Figura 7 se puede observar la gráfica esfuerzo-deformación promedio de los ensayos de compresión realizados a las probetas de aleación 6061 y 6063 respectivamente.

Es importante destacar que las gráficas son diferentes a las producidas por el ensayo de tracción ya que la fractura ocurre a grandes esfuerzos de compresión y esto es debido a que los concentradores de tensiones que puedan estar dentro de la estructura tienden a sellarse por efecto del apilamiento de material. La Figura 10 detalla como las probetas de la aleación 6061 poseen mayor deformación y mayor esfuerzo máximo, los que la hacen más resistentes a los esfuerzos de compresión a diferencia de la curva de la aleación 6063 donde existe menores esfuerzos y deformaciones. Es de destacar la importancia de la deformación como respuesta a una posible fractura, es decir mientras más deformación tenga el material sometido a esfuerzo más posibilidades se tiene para buscar alguna solución a este problema.

Figura 7. Gráfica esfuerzo-deformación promedio del ensayo de compresión de las probetas de aleación 6061 y 6063 respectivamente.

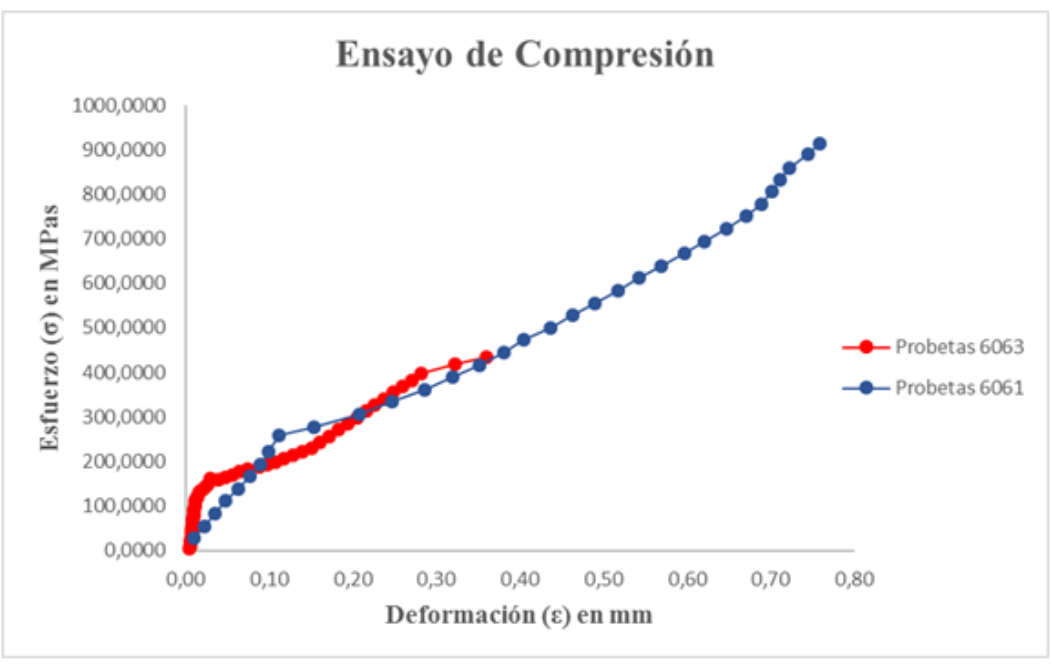


También es importante destacar que el material que será deformado por medio de extrusión debe tener un comportamiento aceptable donde se apliquen esfuerzos considerables haciendo que aumente el endurecimiento por deformación, pero evitar la creación de concentradores de tensiones, todo esto característico de los trabajos en frio.

También se conoce que este endurecimiento disminuye la resistencia a la corrosión por cual desfavorece la cualidad del aluminio como agente principal en el uso de la fabricación de autopartes. Por lo tanto, los resultados de este ensayo de compresión consideran a la aleación 6063 como posible material utilizado para el proceso de fabricación de autopartes ya que tienen menor endurecimiento por deformación haciendo que sea más resistente a la corrosión, la deformación plástica es considerable lo cual puede permitir la extrusión de piezas con diseño moderado mostrado en la Figura 6.

\section{Caracterización microestructural.}

En la Tablas 10 se observa las microestructuras de las aleaciones 6061 y 6063 respectivamente, tomadas a través del microscopio óptico con aumento de 500x y atacadas con HF $2 \%$.

Una de las fases presentes en las aleaciones es el $\mathrm{Mg}_{2} \mathrm{Si}$ la cual proviene de una reacción eutéctica como se muestra en el diagrama de fase de la Figura 34. Este compuesto intermetálico aparece debido a que se agrega en mayor cantidad Si para evitar que el Mg supere el 0,7 y por ende disminuya la velocidad de extrusión. El $\mathrm{Mg}_{2} \mathrm{Si}$ tiene una forma cubica oscura la cual produce endurecimiento por solución sólida porque fija las dislocaciones y por ende la deformación plástica puede ser moderada. Esta fase $\mathrm{Mg}_{2} \mathrm{Si}$ siempre esta acompaña de otra fase la cual es el $\mathrm{Fe}_{3} \mathrm{SiAl}_{12}$ (Metal Handbook Volumen 9, 1985).

El compuesto intermetálico $\mathrm{Fe}_{3} \mathrm{SiAl}_{12}$ esta favorecido en mayor proporción en la aleación 6063 ya que hay mayor composición de Fe y Si haciendo entonces que favorezca su precipitación. También se observa, para ambas aleaciones, que tiene 2 formas. En la aleación 6061 presenta forma poliédrica y es de color gris metálico, pero en la aleación 6063 presenta forma de agujas, característico del tipo widmanstatten.

Este tipo de forma de aguja aumenta la resistencia ya que las dislocaciones presentes en la matriz $\alpha$ chocan contra ellas a diferencia de las poliédricas la cual otorgan un poca más de ductilidad. 
Tabla 10. Microestructuras de la sección transversal de las aleaciones 6061 y 6063 con aumento de 500x, atacadas con HF $2 \%$ y tomadas por medio de microscopio óptico.

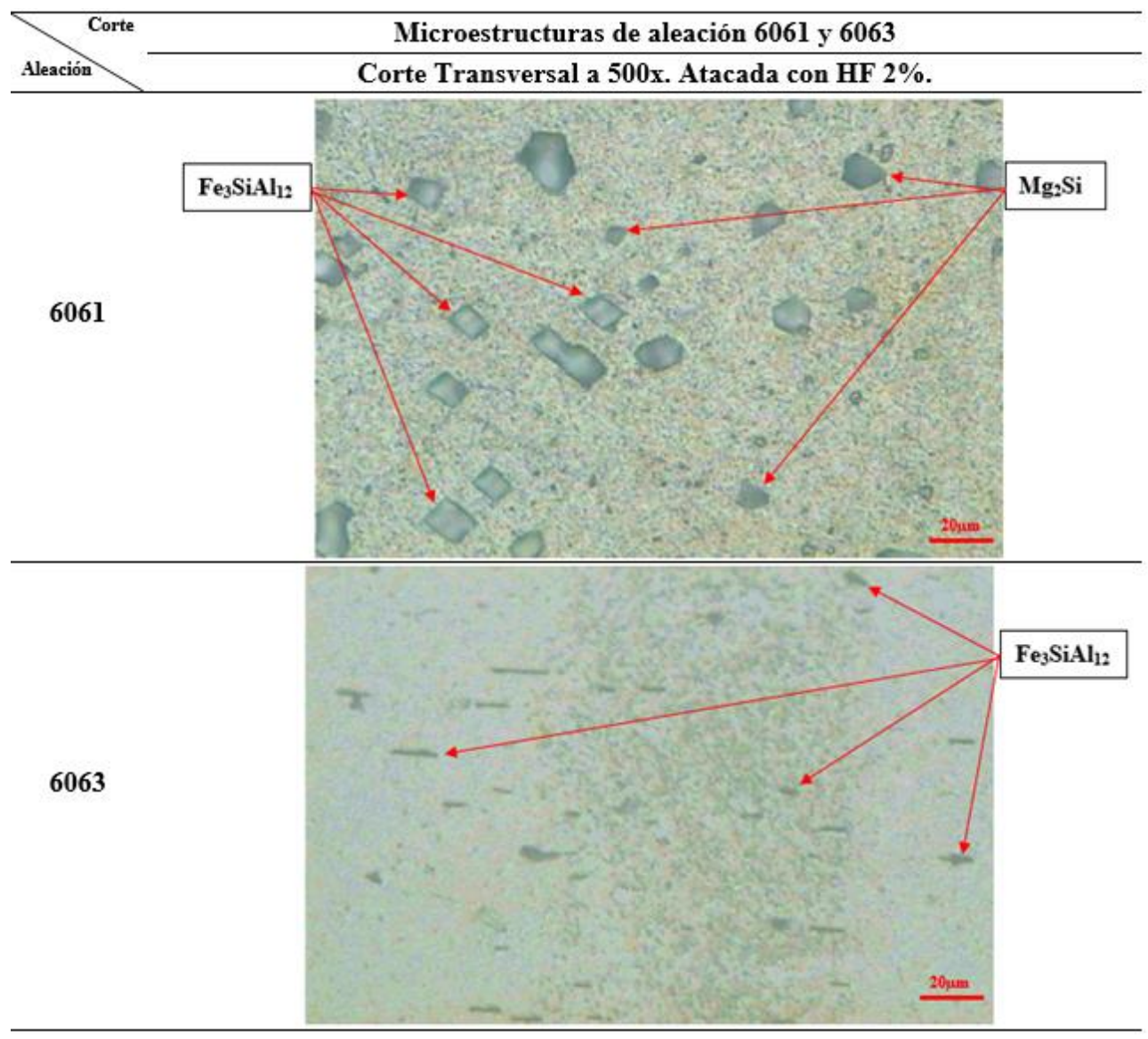

Estos compuestos intermetálicos son los factores principales para modificar las propiedades mecánicas de las aleaciones, pero sobre todo la forma widmanstatten del $\mathrm{Fe}_{3} \mathrm{SiAl}_{12}$ presente en la aleación 6063 ya que genera mayor endurecimiento.

Es aquí donde este compuesto intermetálico $\mathrm{Fe}_{3} \mathrm{SiAl}_{12}$ es el principal causante de los grandes esfuerzos de tracción, grandes durezas y menores esfuerzos de compresión de la aleación 6063 produciendo muy poca deformación plástica.

En la Tabla 11 se observa la microestructura de las aleaciones, 6061 y 6063 respectivamente, provenientes del proceso de extrusión detallado en las líneas y sistemas de producción de autopartes.

Tabla 11. Microestructuras de la sección longitudinal de las aleaciones, 6061 y 6063 respectivamente, deformadas por el proceso de extrusión con aumento del 200x, atacadas con HF 2\% y tomadas por medio de microscopio óptico. 


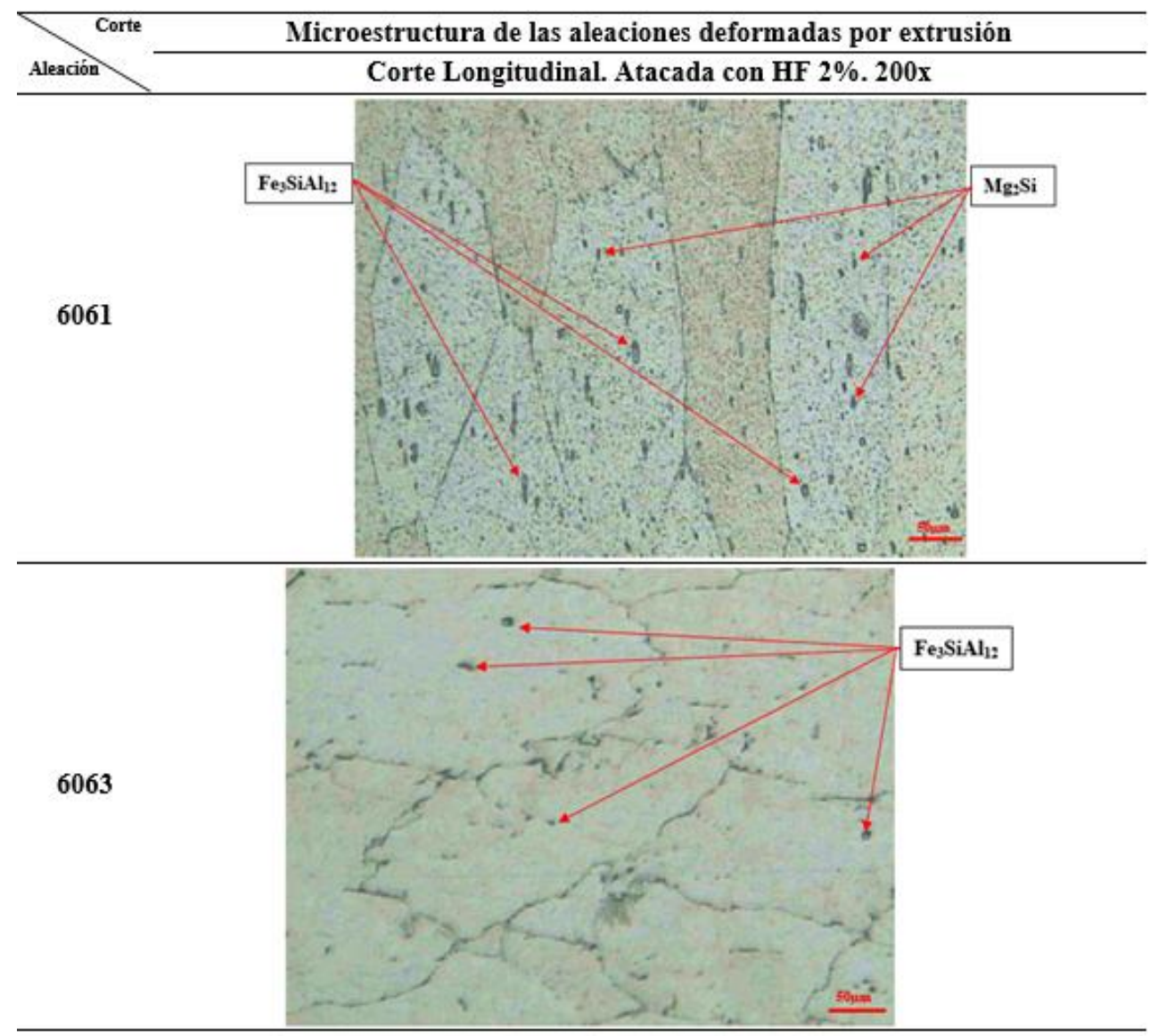

El comportamiento de estos compuestos intermetálicos es diferente después de aplicarse los esfuerzos. En la aleación 6061 ambos presentan una forma alargada producto de la deformación plástica lo cual la hace más vulnerable a los esfuerzos reales dentro del campo de trabajo produciendo, posiblemente una vida útil más corta.

En cambio, en la aleación 6063 los compuestos intermetálicos sufren fractura, en especial el $\mathrm{Fe}_{3} \mathrm{SiAl}_{12}$, haciendo que se dispersen en toda la microestructura. Esto hace que la aleación sea un poco más resistente y pueda soportar los esfuerzos reales dentro de los procesos de conformado de metales.

Cabe destacar que ambas matrices $\alpha$ de las aleaciones 6061 y 6063 presentan alargamiento de grano producto de la deformación plástica del proceso.

\section{Conclusiones.}

- Se realizó un análisis a los sistemas de producción de las empresas dedicadas al proceso de conformado por extrusión de autopartes en la cual arroja que son de transformación ya que necesitan del suministro de materia prima por parte de contratistas. A través de entrevistas a profesionales expertos en el área se determinó 
que hay problemáticas con el desplazamiento de los subproductos entre las estaciones de trabajo por lo cual incrementa el tiempo y aumentan los costos de producción. Es importante resaltar que la productividad está influenciada directamente por las presiones ejercidas por el punzón sobre el tocho hacia la matriz, pero sobre todo por la velocidad de extrusión que a su vez es función de la composición de la aleación. Mientras menor de $0,7 \%$ en peso de $\mathrm{Mg}$, mayor será la velocidad de extrusión y mayor la productividad. Para ambas aleaciones se cumple esta consideración.

- Para solventar la problemática del tiempo de producción se plantea la creación de una línea de producción automatizada después del proceso de troquelado. Estas líneas de producción involucran un gran capital de inversión ya que se deben contar con sistemas de control como PLC, sensores, actuadores, pantallas LCD, pulsadores, cinta transportadora, brazos robóticos, entre otros. Este sistema mecatrónico estará regido por el PLC el cual tendrá comunicación directa con el operador y a su vez recibirá señales de los sensores y dará órdenes a la cinta transportadora o al brazo robótico según sea el caso. Es importante destacar que cada cambio en el sistema de producción debe venir de la mano con los planteamientos exigidos por las normas internacionales, por lo tanto, se sugiere, en dichas organizaciones, implementar sistemas integrados de gestión donde las normas ISO 9000, ISO 14000 y la OHSAS 18000 sean actores principales para obtener autopartes de calidad respetando el medio ambiente y con excelente seguridad y protección laboral.

- Los resultados del ensayo de tracción, compresión y dureza determinaron que la aleación con menos deformación plástica es la 6063 debido a que hay que producir mayores esfuerzos. Esto es consecuencia de la gran cantidad de elementos aleantes que produce un material más resistente.

- Las microestructuras arrojaron la presencia de compuestos intermetálicos dentro de la matriz $\alpha$ de las aleaciones. Estos compuestos $\mathrm{Mg}_{2} \mathrm{Si}$ y $\mathrm{Fe}_{3} \mathrm{SiAl}_{12}$ producen un aumento de la residencia de la aleación, pero la consecuencia más importante es la forma de este precipitado dando como resultado que el $\mathrm{Fe}_{3} \mathrm{SiAl}_{12}$ con forma widmanstatten, presente en la aleación 6063, influye directamente en la resistencia en el material, por lo cual esta aleación tenga mayor endurecimiento que la aleación 6061 ya que este mismo compuesto presenta forma poliédrica haciéndola más dúctil. Esta forma del compuesto explica por qué la aleación produjo mayor resistencia a la tracción y dureza para la aleación 6063. Estas agujas del $\mathrm{Fe}_{3} \mathrm{SiAl}_{12}$ se quebraron después de aplicar la deformación plástica haciendo que se incremente su resistencia a trabajos reales en el uso diario de la pieza.

- Por lo tanto, se recomienda escoger la aleación 6063 como posible material utilizado para el proceso de fabricación de autopartes ya que se aplican menores esfuerzos de compresión, evitando que se pierda la resistencia a la corrosión, sin importar la deformación plástica ya que el diseño de las piezas es moderado. Además, 
se produce mayor resistencia después del proceso de conformado debido a las formas del $\mathrm{Fe}_{3} \mathrm{SiAl}_{12}$ después de la deformación incrementando así la vida útil de la autoparte.

\section{Referencias bibliográficas.}

Bardellini, J. (2005). Análisis del sector automotor ecuatoriano. Apunte de Economía, 1-52.

Callister, W. (2014). Introducción a la ciencia e ingeniería de los materiales. Barcelona, España: Editorial Reverté, S.A.

Cuatrecasas, L. (2011). Gestión económica de la producción. Madrid, España: Díaz de Santos.

Iplacex. (29 de Mayo de 2018). Biblioteca Iplacex. Obtenido de http://biblioteca.iplacex.cl/RCA/Tipos\%20sistemas\%20de\%20producción.pdf

James, B. (29 de 05 de 2018). Automotive manufacturing solutions. Obtenido de http://www.automotivemanufacturingsolutions.com/es/proceso-materiales/elaluminio-en-la-industria-del-automovil

Metal Handbook Volumen 2, A. (1989). Properties and Selection: Nonferrous Alloys and Special-Purpose Materials. Estados Unidos: ASM HANDBOOK.

Metal Handbook Volumen 9, A. (1985). Metallography and Microstructures. Estados Unidos: ASM Metal Hand Books.

Nañez, M. (1998). Comportamiento de una aleación de aluminio tratada termicamente bajo carga de compresión. Nuevo León, México: Universidad Autonoma de Nuevo León.

Navas, H., Vaca, W., Nuñez, D., Paredes, J., \& Morales, F. (2017). Análisis cuantitativo de los elementos aleantes principales precipitados en billets de aluminio de aleación 6063 en equipo de fundición de colada continua horizontal para el proceso de extrusión. Ingenius, 42-50.

Sipper, D., \& Bulfin, R. (1998). Planeación y Control de la Producción. Ciudad de México, México: McGraw-Hill Interamericana Editores, S. A.

TALAT 1302, H. (1994). Aluminium Extrusion: Alloys, Shapes and Properties. Birmingham, Inglaterra: Aluminium Federation.

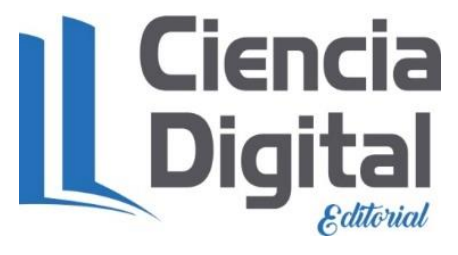




\section{Para citar el artículo indexado.}

Chica L., Rocha J., Martínez J. \& Cabascango P. (2019) Análisis del sistema de producción y caracterización microestructural para evaluar el comportamiento mecánico de chasis de vehículo fabricado por extrusión. Revista electrónica Ciencia Digital 3(1), 461-480. Recuperado desde: http://cienciadigital.org/revistacienciadigital2/index.php/CienciaDigital/article/view/349/76 $\underline{8}$

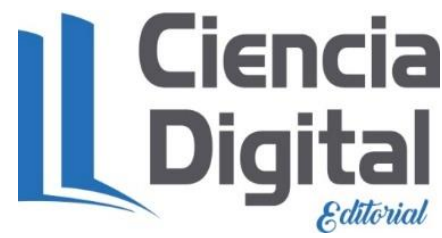

El artículo que se publica es de exclusiva responsabilidad de los autores y no necesariamente reflejan el pensamiento de la Revista Ciencia Digital.

El artículo queda en propiedad de la revista y, por tanto, su publicación parcial y/o total en otro medio tiene que ser autorizado por el director de la Revista Ciencia Digital.
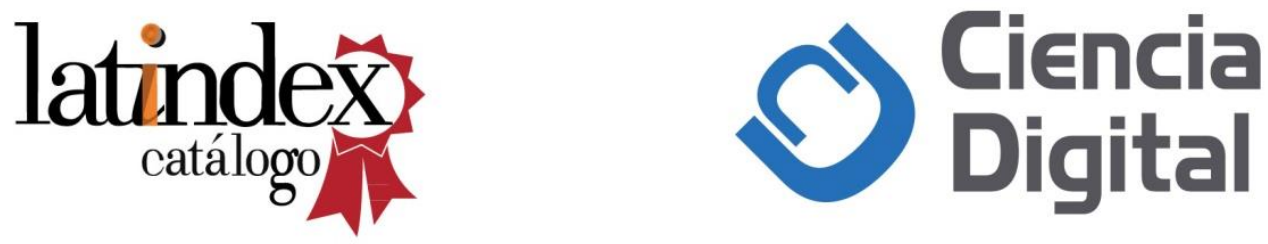